\title{
A METHOD FOR DETERMINATION OF SPECIFIC ELECTRICAL RESISTANCE OF STEEL AND NANO-COATING SPUTTERED ON IT
}

\section{Andris Martinovs ${ }^{1}$, Josef Timmerberg ${ }^{2}$, Konstantins Savkovs ${ }^{3}$, Aleksandrs Urbahs ${ }^{3}$, Paul Beckmann ${ }^{2}$}

1- Rezeknes Augstskola, Atbrivosanas aleja 90, Rezekne, LV 4601, Latvia

2- Jade Hochschule, Friedrich-Paffrath-Straße 101, 26389, Wilhelmshaven, Germany

3- Riga Technical University, Institute of Transport Vehicle Technologies

Lomonosova 1/V, Riga, LV 1019, Latvia

E-mail: ${ }^{1}$ Andris.Martinovs@ @u.lv, ${ }^{2}$ jt@jade-hs.de, ${ }^{3}$ Konstantins.Savkovs@ @rtu.lv,

${ }^{4}$ Aleksandrs.Urbahs@rtu.lv, ${ }^{5}$ beckmann@jade-hs.de

\begin{abstract}
The paper describes methods developed to determine specific electrical conductivity and relative magnetic permeability of cylindrical steel items and nano-coatings deposited on them by sputtering. Research enables development of a new method for determination of thickness of vacuum deposited nanocoating that is based on application of skin effect.
\end{abstract}

Keywords: electrical resistance, magnetic permeability, nano-coating thickness, skin effect, steel.

\section{Introduction}

Development and implementation of technologies of sputtered protective nano-coatings require control over a thickness of the coatings which usually vary between $0.5-5 \mu \mathrm{m}$. Traditionally, the thickness is determined using a scanning optical or electron microscope that implies destruction of a sample, it has to be sawn, polished, etched and then measured. Authors' previous experience tells that a preparation of one sample takes $2-3$ hours. It is necessary to develop less time consuming methods. The coatings contain metals; increase in the thickness also increases electrical conductivity. Therefore, the determination of the thickness could be based on electrical conductivity measurements. However, this approach has problems because steel is good electrical conductor, its resistance is comparable to that of electrical contacts and leads, the thickness of the coating is small in comparison to a size of a coated part and impact of the coating on total electrical conductivity of the part is negligible. The solution could be based on skin effect: at sufficiently high frequencies electrical charge only flows through a thin layer on the top of a conductor, electrical conductivity can become significant and can be measured. Therefore impact of the coating on total electrical conductivity can be significant and its value can be indicative of the thickness of the coating. To determine it, electrical and magnetic curve values have to be known. [1], [2], [3], [4]

The purpose of the paper is to develop methods for determination of specific electrical conductivity and relative magnetic permeability of steel and a nano-coating for electrical measuring of nano-coating thickness.

\section{Determination of the specific electrical conductivity}

Materials and Methods

Experiments are performed using a steel tube with length $l=153 \mathrm{~mm}$ and inside/outside diameter $d_{1} / d_{2}=6 / 8 \mathrm{~mm}$. To ensure good electrical contact, copper wires are soldered to both ends of the tube. HP $4284 \mathrm{~A} 20 \mathrm{~Hz}-1 \mathrm{MHz}$ LCR-meter is used to measure electrical resistance. The electrical resistance of the tube is

$$
R=\frac{l}{\kappa \cdot A},
$$

where $\kappa$ is specific electrical conductivity, $A-$ cross-section of charge flow area. 
If high frequency $f$ alternate current is charged through the tube, the depth of the skin effect [1], [2]

$$
\delta=\frac{1}{\sqrt{\pi \cdot \mu_{0} \cdot f \cdot \kappa \cdot \mu}},
$$

where $\mu$ - relative magnetic permeability of steel, $\mu_{0}=4 \pi \cdot 10^{-7} \mathrm{H} / \mathrm{m}$ - magnetic constant.

In this case we can assume that the charge flows only through outer layer of the tube with depth $\delta$ and density of the current is the same in all points of this layer. Cross sectional area of the charge flow (represented in grey in Figure 1)

$$
A=\pi \cdot\left(d_{2}-\delta\right) \cdot \delta
$$

This equation is valid if $\delta \leq\left(\mathrm{d}_{2} / 2-\mathrm{d}_{1} / 2\right)$. From expressions (1) - (3) follows

and

$$
R=\frac{l}{d_{2} \cdot \sqrt{\frac{\pi \cdot \kappa}{\mu_{0} \cdot \mu \cdot f}}-\frac{1}{\mu_{0} \cdot \mu \cdot f}}
$$

$$
\kappa=\frac{\mu_{0} \cdot \mu \cdot f}{\pi \cdot d_{2}^{2}} \cdot\left(\frac{l}{R}+\frac{1}{\mu_{0} \cdot \mu \cdot f}\right)^{2} .
$$

To use equation (5), we need to know electric resistance $\mathrm{R}$ only for the tube excluding resistance of the contacts and the leads. To find that, one input of the meter is connected to the copper wire soldered to the end of the tube, but the other input - to a crocodile clamp that holds the tube. Total electrical resistance $\mathrm{R}_{\mathrm{k}}$ of the tube is measured in different sections with length from 0 to $l$ at the constant frequency $f$. The acquired curve $\mathrm{R}_{\mathrm{k}}=\mathrm{R}_{\mathrm{k}}(l)$ allows to determine electrical resistance of the contacts and the leads (it is $l=0$ ).

\section{Determination of the magnetic permeability of steel}

In order to calculate $\kappa$, we have to know relative magnetic permeability of steel $\mu$ (see equation (5)). To determine it, solenoid coil with length $l$, diameter $\mathrm{D}<<l$, number of turns $\mathrm{N}$ is used. The solenoid is wound on a dielectric tube, it does not have a core and its inductance is $\mathrm{L}_{1}$ (see Figure 2). The steel tube used in the experiments is inserted in the solenoid. The tube has the same length as (or is a little longer than) the solenoid (see Figure 3). As relative magnetic permeability of steel $\mu>>1$, inductance of the solenoid in this case is $L_{2}>L_{1}$.

Inductance of the solenoid without a core is:

$$
L_{1}=\frac{\mu_{0} \cdot \pi \cdot N^{2} \cdot D^{2}}{4 \cdot l} .
$$

System, where solenoid and the tube inserted in it has the same length, can be considered parallel magnetic circuit with magnetic flux

$$
\Phi=\Phi_{1}+\Phi_{2}=B_{1} \cdot A_{1} \cdot N+B_{2} \cdot A_{2} \cdot N
$$

where $\Phi_{1}$ - magnetic flux in the tube, $\Phi_{2}$ - magnetic flux in free space inside the solenoid. Using Ampere's circuital law, we can determine that inductance of a steel tube placed in long solenoid $(l>>D)$ is

$$
B_{1}=\frac{\mu \cdot \mu_{0} \cdot I \cdot N}{l}
$$

and in free space within solenoid

$$
B_{2}=\frac{\mu_{0} \cdot I \cdot N}{l},
$$

where I - current passing through loops. 
Cross sectional area of the steel tube (perpendicular to the longitudinal axis of the solenoid):

$$
A_{1}=\frac{\pi \cdot d_{2}^{2}}{4}-\frac{\pi \cdot d_{1}^{2}}{4} \text {. }
$$

Cross sectional area of the free space (perpendicular to the longitudinal axis of the solenoid):

$$
A_{2}=\frac{\pi \cdot D^{2}}{4}-\left(\frac{\pi \cdot d_{2}^{2}}{4}-\frac{\pi \cdot d_{1}^{2}}{4}\right)
$$

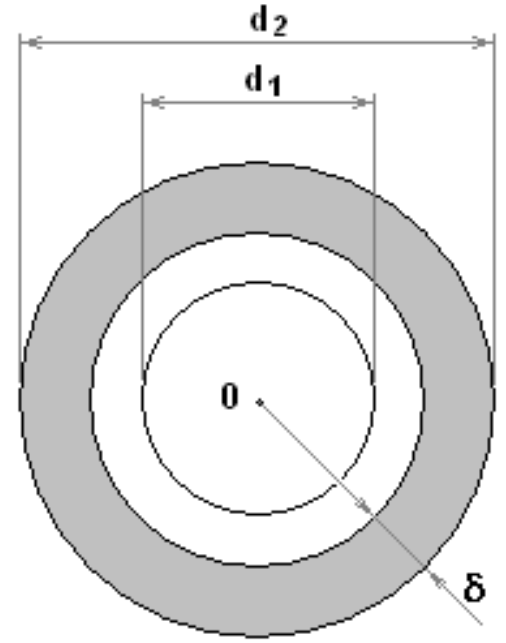

Fig.1. Cross sectional area of the tube with high frequency current flowing through it

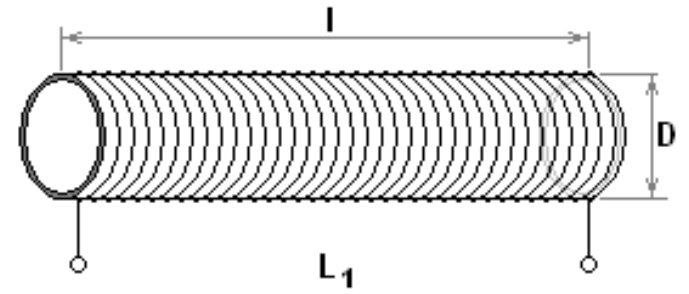

Fig.2. Solenoid without a core

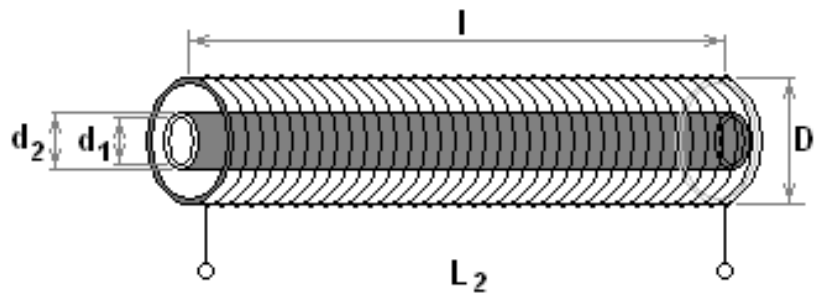

Fig.3. Solenoid with the steel tube inserted in it

Placing last four expressions in the equation (7), taking to account expression (6) and relation between magnetic flux $\Phi$, inductance $\mathrm{L}$ and current through solenoid I

$$
\Phi=L \cdot I
$$

We obtain inductance $\mathrm{L}_{2}$ of the solenoid with the steel tube as a core is:

$$
L_{2}=\frac{\mu_{0} \cdot \pi \cdot N^{2} \cdot\left(d_{2}^{2}-d_{1}^{2}\right)}{4 \cdot l} \cdot(\mu-1)+L_{1} .
$$

It follows that relative magnetic permeability of steel is

$$
\mu=\frac{4 \cdot l \cdot\left(L_{2}-L_{1}\right)}{\mu_{0} \cdot \pi \cdot N^{2} \cdot\left(d_{2}^{2}-d_{1}^{2}\right)}+1 .
$$

The steel tube described in the section 2.1. is used in the experiments. Inductance is measured with Peak Tech 2010 DMM multimeter. Before electrical measurements are taken, the tube is heated to $800^{\circ} \mathrm{C}$ that exceeds Curie temperature for iron $\left(768^{\circ} \mathrm{C}\right)$. For this reason steel does not have lasting magnetization. During inductance measuring process, steel is not magnetized, therefore obtained $\mu$ value conforms to intensity of magnetic field $\mathrm{H}=0$. Five solenoid coils of different length, diameter and number of turns are used for measurements.

To eliminate measuring errors, the method of partial derivation is applied. According to it, absolute error of relative magnetic permeability $\mu$ is

$$
\Delta \mu=\sqrt{\left(\frac{\partial \mu}{\partial l} \cdot \Delta l\right)^{2}+\left(\frac{\partial \mu}{\partial L_{1}} \cdot \Delta L_{1}\right)^{2}+\left(\frac{\partial \mu}{\partial L_{2}} \cdot \Delta L_{2}\right)^{2}+\left(\frac{\partial \mu}{\partial d_{1}} \cdot \Delta d_{1}\right)^{2}+\left(\frac{\partial \mu}{\partial d_{2}} \cdot \Delta d_{2}\right)^{2}},
$$

where $\Delta l$ - error of tube length measurement $(0.5 \mathrm{~mm}) ; \Delta \mathrm{L}_{1}$ and $\Delta \mathrm{L}_{2}$ - measurement error of inductance of solenoid without a core and with the steel tube inserted $(1 \mu \mathrm{H}) ; \Delta \mathrm{d}_{1}$ and $\Delta \mathrm{d}_{2}-$ measurement error of inside and outside diameter $(0.1 \mathrm{~mm})$ of the steel tube. 
The partial derivations in the equation (15) are obtained by derivation of the expression (14). Absolute error of specific electrical conductivity $\kappa$ is:

$$
\Delta \kappa=\sqrt{\left(\frac{\partial \kappa}{\partial \mu} \cdot \Delta \mu\right)^{2}+\left(\frac{\partial \kappa}{\partial f} \cdot \Delta f\right)^{2}+\left(\frac{\partial \kappa}{\partial d_{2}} \cdot \Delta d_{2}\right)^{2}+\left(\frac{\partial \kappa}{\partial l} \cdot \Delta l\right)^{2}+\left(\frac{\partial \kappa}{\partial R} \cdot \Delta R\right)^{2}},
$$

where $\Delta \mathrm{f}$ - frequency measurement error $(10 \mathrm{~Hz}) ; \Delta \mathrm{R}$ - measurement error of electrical resistance of the tube $(0.01 \Omega)$. Partial derivations of equation (16) are obtained by derivation of the expression (5).

\section{Determination of specific conductivity of sputtered nano-coating}

On the outside surface of the tube used in the above experiments Al-Ti-N nano-coating is deposited by sputtering in vacuum using electric arc and magnetron (equipment HНB-66-И1, deposition time $30 \mathrm{~min}$ ). At the same time Al-Ti-N coating is deposited also on dielectric film (one surface) that is placed in maximum proximity to the steel tube in a vacuum chamber. For the first approximation it is assumed that composition, structure and specific electric conductivity of the coating on the tube and on the dielectric film are the same. Rectangular slip sized $200 \mathrm{~mm}$ by $12.5 \mathrm{~mm}$ is cut out of the film and secured with crocodile clamps to the HP 4284A LCR meter. Specific electrical conductivity of the coating is

$$
\kappa_{2}=\frac{l}{R \cdot b \cdot h},
$$

where $l$ - length of the slip, width of the slip $(12.5 \mathrm{~mm}), \mathrm{h}-$ thickness of the coating, $\mathrm{R}-$ electrical resistance.

\section{Results and Discussion}

Applying the method described in section 2.2., relative magnetic permeability $\mu=27.9 \pm 2.0$ is determined for the particular type of steel. The comparatively small value of relative magnetic permeability of the steel is explained by a fact that before measurements were taken, the sample was heated above Curie temperature and as a result it does not have lasting magnetisation. Steel items are subjected to such heating during deposition of protective coating.

Equipment used to construct the curve depicting resistance-frequencies of the tube is shown in Figure 4 but the curve itself - in Figure 5. Resistance R values include electrical resistance of the tube, soldering, the copper leads and contacts that secure leads to the equipment. To determine electrical resistance of the tube, we measure resistance in its different sections with length 0 to $153 \mathrm{~mm}$ at the frequency $1 \mathrm{MHz}$ (see Fig. 6). The dependency of the resistance on the length of a section is shown in Figure 7. The total resistance of the tube with leads and contacts is $0.1141 \Omega$ (if $l=153 \mathrm{~mm}$ ), but resistance of leads and contacts is $0.0498 \Omega$ (if $l=0$ ), that constitutes $43.6 \%$ of total value of the resistance. The resistance of the $153 \mathrm{~mm}$ tube (without the leads and contacts) is $0.0643 \Omega$. Assuming, that for this particular sample the proportion $(43.6 \%)$ between resistance of the leads/contacts and the total resistance stays the same at different frequencies of alternating current, the curve shown in Figure 5 is recalculated excluding the resistance of the leads and contacts. Results of the calculations (points) and their margins of error are shown in Figure 8.

Placing at $1 \mathrm{MHz}$ acquired resistance of the tube $\mathrm{R}=0.0643 \Omega$ in the equation (5), we calculate specific electrical conductivity for given type of steel: $\kappa_{1}=(1.01 \pm 0.32) \cdot 10^{6} \mathrm{~S} / \mathrm{m}$. 


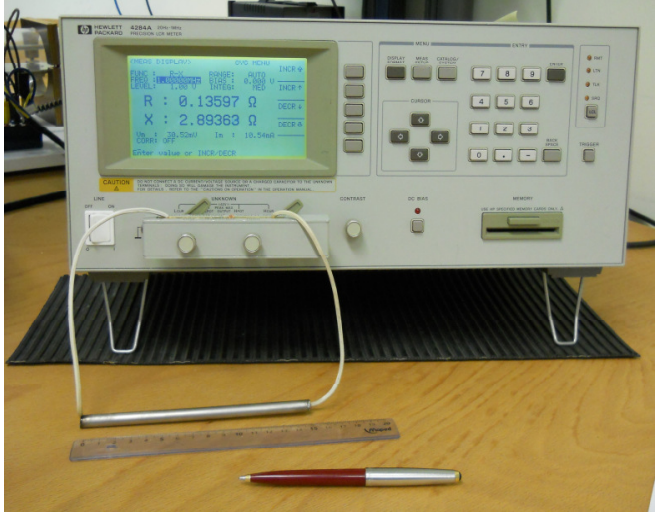

Fig.4. HP 4284A 20Hz- 1MHz LCR meter with steel tube connected to it

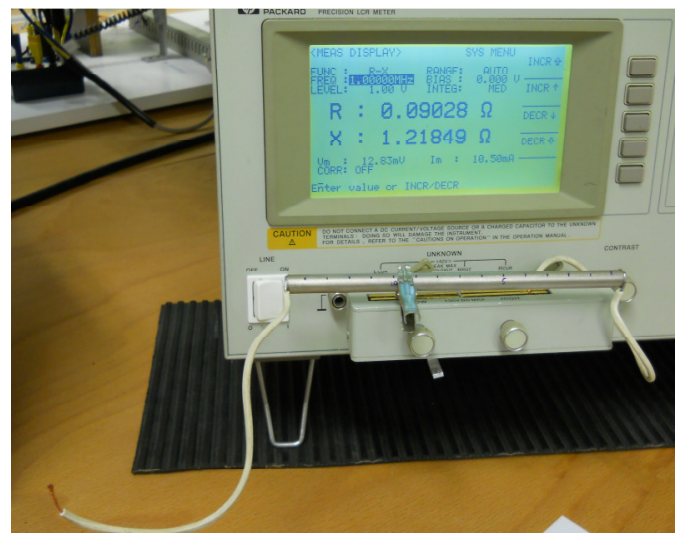

Fig.6. HP 4284A 20Hz- 1MHz LCR meter with the connected steel tube, resistance of the $10 \mathrm{~cm}$ long section is measured

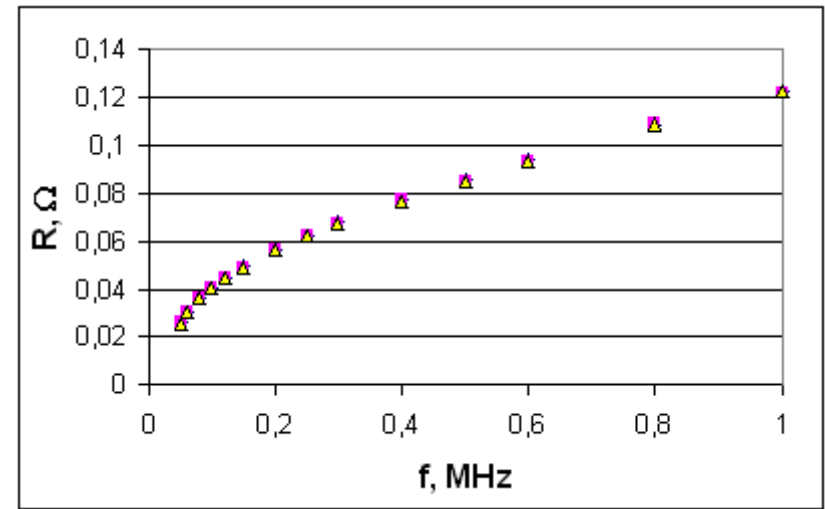

Fig.5. Curve of electrical resistance frequencies of the steel tube with leads and contacts

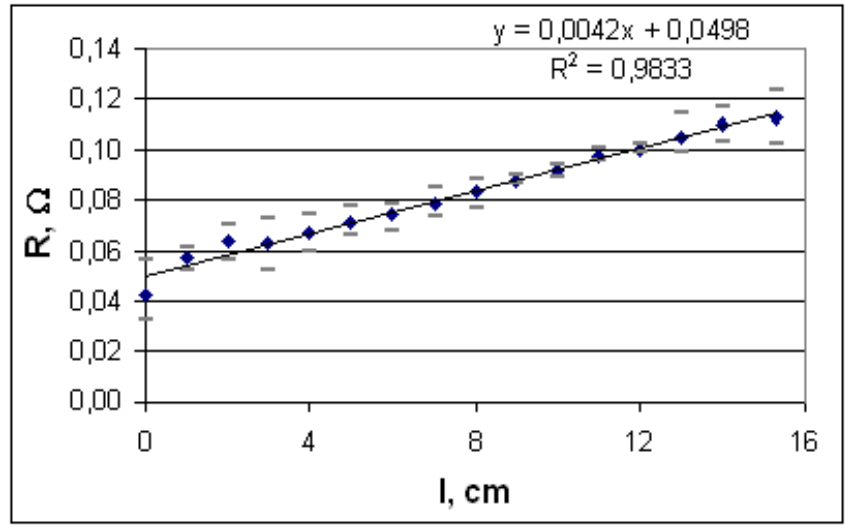

Fig.7. Electrical resistance (at $1 \mathrm{MHz})$ depending on the length of the section

Knowing $\kappa$ and $\mu$ for particular steel and using the equation (4), the curve of resistance frequencies can be obtained. In the Figure 8 it is shown as unbroken curve. Within margins of error it coincides with experimental data. This confirms that the model described in section 2 is right.

Using SEM HITACHI S-3000N equipped with BRUKER $133 \mathrm{eV}$ spectrometer, chemical compositions of the coatings on the steel tube (Table 1; Fig. 9) and the dielectric film (Table 2, Fig. 12) are determined. Comparing data from the tables, we conclude that chemical composition of titanium and nitrogen in both coatings is within measuring margins of error but composition of aluminum - differs. Since amount of aluminum is not big, we can assume that the chemical compositions of both coatings are approximately the same and the values of specific electrical conductivity $\kappa_{2}$ are also approximately the same. It is no exactly precise because during deposition process the steel tube is connected to negative electrical potential that in vacuum promotes positive metal and nitrogen ions reaching surface of the steel; the surface of dielectric film does not have such potential. Before deposition process the surface of the steel is subjected to ion bombardment that cleanses and heats it up to $800^{\circ} \mathrm{C}$ improving quality of sputtered layer; it is not possible to perform this process with dielectric film. Therefore the composition, structure and properties of the coatings deposited on surfaces of the steel tube and dielectric film can vary. 


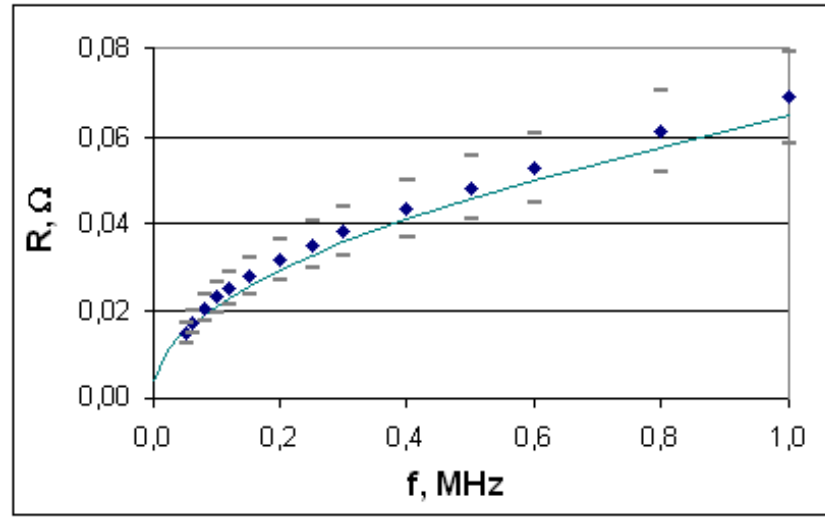

Fig.8. The curve of electrical resistance frequencies of the steel tube (without the leads and contacts)

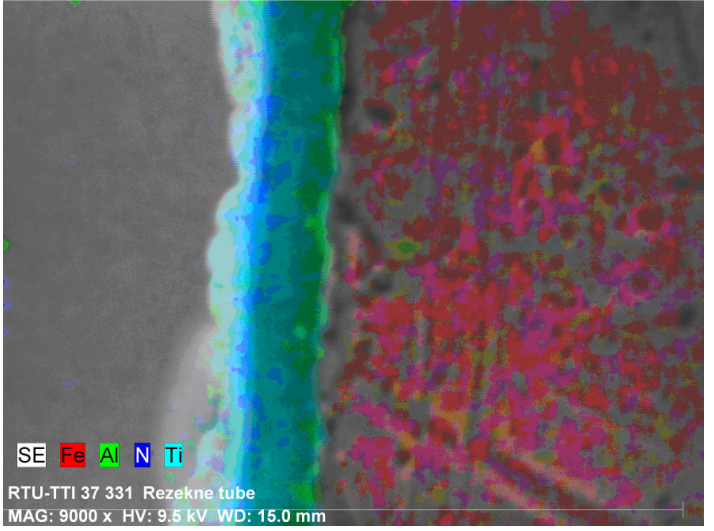

Fig.9. Chemical composition of the nanocoating Al-Ti-N deposited on the steel tube; thickness of the coating $1.6 \mu \mathrm{m}$

Table 1.

Table 2.

Composition of nano-coating deposited on the steel tube

\begin{tabular}{|c|c|c|}
\hline & Mass \% & Atoms \% \\
\hline $\mathrm{N}$ & $53,91 \pm 17$ & $77,89 \pm 14$ \\
\hline $\mathrm{Al}$ & $2,49 \pm 1,3$ & $2,06 \pm 1,6$ \\
\hline $\mathrm{Ti}$ & $43,59 \pm 16$ & $20,05 \pm 12$ \\
\hline
\end{tabular}

Composition of nano-coating deposited on the dielectric film

Dependence of electrical resistance of the dielectric film coating on the length of the slip $l$ (see Fig. 10 and 11) is measured at frequency 1MHz. Difference in resistance measurements can be explained with a fact that coating do not adhere well to the dielectric film used in the experiments; it suffers local damage every time the crocodile clamps are attached, it is not possible to create same electrical contact between the coating and the crocodile clamps. It is indicated by tendency that shows increased electrical resistance with every repeated measurement.

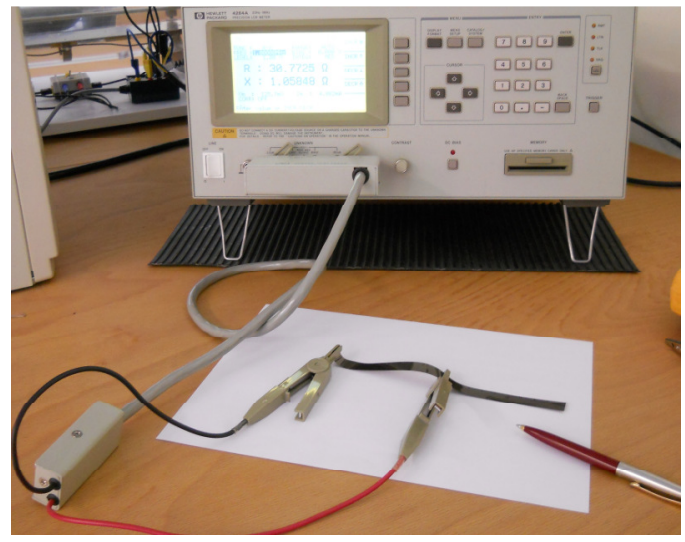

Fig.10. Measuring electrical resistance of Al-Ti-N nano-coating deposited on the dielectric film

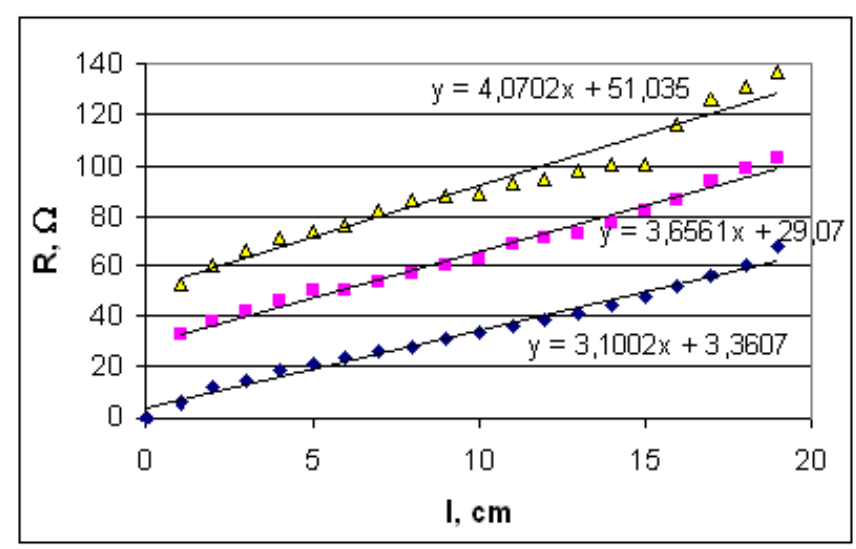

Fig. 11. Electrical resistance of Al-Ti-N nano-coating deposited on the dielectric film (at frequency $1 \mathrm{MHz}$ ) depending on the length of the section of the slip 


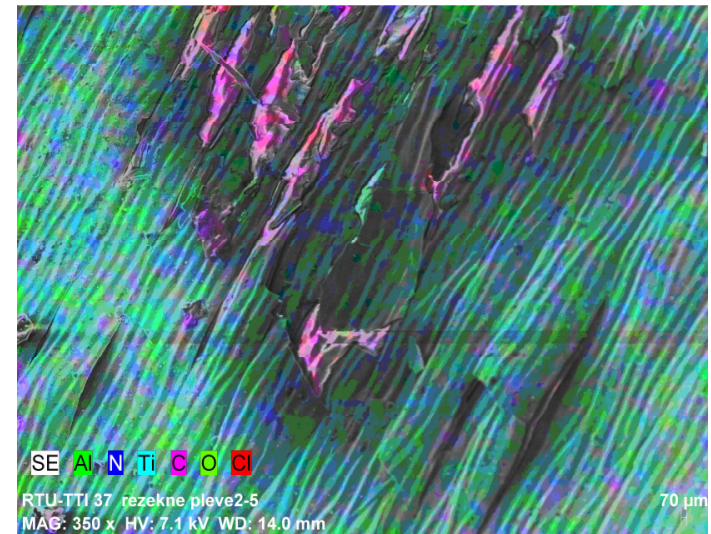

Fig. 12. Mechanically damaged Al-Ti-N nano-coating on the dielectric film; colors indicate its chemical composition

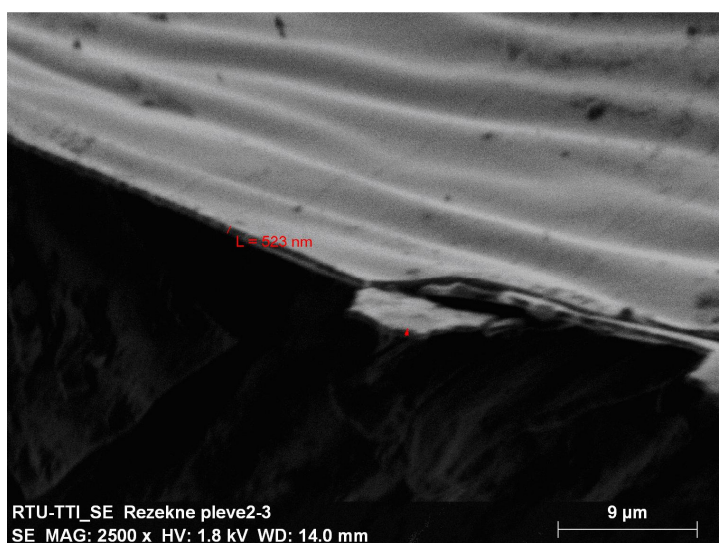

Fig. 13. Fragment of the Al-Ti-N nanocoating that has separated from the surface of the dielectric film; its thickness is $523 \mathrm{~nm}$

Due to the poor adherence of the coating to the dielectric film, we cannot use traditional methods to measure thickness of the coating - polishing an edge of the film and measuring the thickness with a scanning electron microscope. The different solution is offered to take measurements: the coating on the dielectric film is mechanically damaged (see Fig. 12); then separated fragments of the coating with an edge towards an electron beam are located and their thickness measured (see Fig. 13). Statistically processing data from Figure 11, using the equation (17) and measured value of the thickness $h=523 \mathrm{~nm}$, the specific electrical conductivity of the given Al-Ti-N nano-coating is determined $\kappa_{2}=(0,43 \pm 0,11) \cdot 10^{6} \mathrm{~S} / \mathrm{m}$.

The scanning electron microscope is used to measure the thickness of the nano-coating deposited on the steel tube: that is $1.6 \mu \mathrm{m}$ (see Fig. 9). We conclude that simultaneously depositing coating on steel and dielectric surfaces, thickness of the coatings will be different (up to 3 times).

\section{Conclusions}

1. The method to experimentally determine specific electrical conductivity using high frequency current for steel (and other conductors) is developed. The method to determine relative magnetic permeability of steel is developed. It is established that steel used in the experiments has specific electrical conductivity $\kappa_{1}=(1,01 \pm 0,32) \cdot 10^{6} \mathrm{~S} / \mathrm{m}$ and relative magnetic permeability $\mu=27,9 \pm 2,0$.

2. Developed first approximation method for determination of the specific electrical conductivity of the nano-coatings. The specific electrical conductivity of the Al-Ti-N nano-coating deposited on the dielectric film is $\kappa_{2}=(0,43 \pm 0,11) \cdot 10^{6} \mathrm{~S} / \mathrm{m}$.

3 . The research offers opportunity to develop methods for measuring thickness of nanocoatings deposited on cylindrical conductors that are based on electrical measurements.

\section{Acknowledgment}

This work has been supported by the Latvian Council of Science within the projects "Development of technology for the creation of multicomponent nanostructured protective coatings for industrial products" Nr.10.0009.

\section{References}

1. Einfürung in die Feldtheorie. Herausgeber: Arnim Nethe, Hanns-Dietrich Stahlmann, Verlag Dr.Köster, Berlin, 2003.

2. L.Hannakam. Einfürung in die Feldtheorie, TU Berlin, 1974, Vorlesungs- Niederschrift.

3. J. D. Jackson, Classical Electrodynamics (John Wiley \& Sons, Inc., New York, NY, 2004), 3nd ed.

4. K. Simonyi. Theoretische Elektrotechnik. VEB Deutscher Verlag der Wissenschaften, Berlin, 1989. 